\title{
Error Analysis of Satellite Precipitation Products in Mountainous Basins
}

\author{
Yiwen Mei And Emmanouil N. Anagnostou \\ Civil and Environmental Engineering, University of Connecticut, Storrs, Connecticut \\ EFTHYMIOS I. NiKOLOPOULOS AND MARCO BORGA \\ Department of Land, Environment, Agriculture and Forestry, University of Padova, Legnaro, Padua, Italy
}

(Manuscript received 22 November 2013, in final form 17 April 2014)

\begin{abstract}
Accurate quantitative precipitation estimation over mountainous basins is of great importance because of their susceptibility to hazards such as flash floods, shallow landslides, and debris flows, triggered by heavy precipitation events (HPEs). In situ observations over mountainous areas are limited, but currently available satellite precipitation products can potentially provide the precipitation estimation needed for hydrological applications. In this study, four widely used satellite-based precipitation products [Tropical Rainfall Measuring Mission (TRMM) Multisatellite Precipitation Analysis (TMPA) 3B42, version 7 (3B42V7), and in near-real time (3B42-RT); Climate Prediction Center (CPC) morphing technique (CMORPH); and Precipitation Estimation from Remotely Sensed Imagery Using Artificial Neural Networks (PERSIANN)] are evaluated with respect to their performance in capturing the properties of HPEs over different basin scales. Evaluation is carried out over the upper Adige River basin (eastern Italian Alps) for an 8-yr period (2003-10). Basin-averaged rainfall derived from a dense rain gauge network in the region is used as a reference. Satellite precipitation error analysis is performed for warm (May-August) and cold (September-December) season months as well as for different quantile ranges of basin-averaged precipitation accumulations. Three error metrics and a score system are introduced to quantify the performances of the various satellite products. Overall, no single precipitation product can be considered ideal for detecting and quantifying HPE. Results show better consistency between gauges and the two 3B42 products, particularly during warm season months that are associated with high-intensity convective events. All satellite products are shown to have a magnitude-dependent error ranging from overestimation at low precipitation regimes to underestimation at high precipitation accumulations; this effect is more pronounced in the CMORPH and PERSIANN products.
\end{abstract}

\section{Introduction}

Measuring surface rainfall is of great importance; particularly for the heavy precipitation events (HPEs) occurring over mountainous regions that often act as flash flood-triggering storms (Borga et al. 2010). Methods for quantifying precipitation include ground observations from rain gauge and weather radar networks, estimates inferred from satellite observations, outputs from numerical weather prediction models, and estimates produced by a combination of all these different products (Michaelides et al. 2009). Each of these

Corresponding author address: Emmanouil N. Anagnostou, CEE, University of Connecticut, 261 Glenbrook Rd., Unit 3037, Storrs, CT 06269.

E-mail: manos@engr.uconn.edu is associated with specific rainfall estimation uncertainties. Rain gauge networks are the most common estimation method. These networks can provide accurate pointwise precipitation measurements, but the spatial representativeness is limited (Anagnostou et al. 2010; Sapiano and Arkin 2009). Weather radar networks provide precipitation estimates with high spatial and temporal resolutions (i.e., $1-4 \mathrm{~km}$ and $5-15 \mathrm{~min}$ ) but with variable accuracy. Moreover, mountainous terrain tends to degrade the accuracy of radar-derived rainfall estimates because of observational limitations (beam blockages and ground clutter) and their interaction with precipitation vertical structure (Ciach et al. 2007; Germann et al. 2006; Piccolo and Chirico 2005; Anagnostou et al. 2004; Sharif et al. 2002). In addition, radar observations have limited utility in cold weather, when the beam detects primarily snow, which complicates the assessment 
of surface precipitation (Schneebeli et al. 2013). Combining rain gauges with weather radar gives a partial solution to the accuracy issues, but it is not a viable solution for cases with large radar beam blockages due to orography or in areas where those systems are not widely available.

Satellite-based estimates of precipitation can potentially provide a solution to the spatial sampling limitations of ground-based sensors. Satellite sensors are uninhibited by mountains and provide global coverage without spatial inconsistencies (Sapiano and Arkin 2009; Kidd et al. 2003; Scofield and Kuligowski 2003; Arkin and Ardanuy 1989). Several of the current global-scale satellite precipitation retrieval algorithms are based on the combination of high-spatiotemporal-resolution observations in the visible-infrared (VIS-IR) spectrum from geostationary (GEO) satellites and the less frequent but more direct precipitation observations from active and passive microwave (MW) sensors deployed on low-Earth-orbiting (LEO) satellites. The VIS-IR techniques relate surface precipitation to cloud-top information (brightness temperatures) with a high sampling frequency (15-min/3-4-km resolution, 1-km VIS). However, these measurements cannot directly retrieve surface precipitation from the inferred cloud-top properties, implying a weak link between cloud-top information and surface precipitation estimation (Sapiano and Arkin 2009). On the other hand, MW techniques are more accurate than the VIS-IR because they physically link the signal received by the satellite sensors to the size and phase of the hydrometeors present within the observed atmospheric column. Nonetheless, MW observations are associated with a large degree of sampling error, particularly in dealing with short rain events because of their low observational frequency and largesensor field-of-view areas (Ebert et al. 2007; Kidd et al. 2003).

It is deemed by many studies that rainfall retrieved from either VIS-IR or MW sensors, or the combination of both sensor observations, suffers from noticeable deficiencies compared to ground-based measurements (Stampoulis and Anagnostou 2012; AghaKouchak et al. 2011; Fleming et al. 2011; Yong et al. 2010; Su et al. 2008; Dinku et al. 2007; Ali et al. 2005). Stampoulis and Anagnostou (2012) conducted an analysis for Tropical Rainfall Measuring Mission (TRMM) Multisatellite Precipitation Analysis (TMPA) 3B42 product, version 6 (3B42-V6), and the Climate Prediction Center (CPC) morphing technique (CMORPH) over continental Europe and found that correlations of the two rainfall products to the gauge-interpolated rainfall are magnitude dependent in terms of daily rainfall accumulation; moreover, the products exhibited more pronounced seasonal dependency over high-elevation regions compared to the low-elevation areas. Anagnostou et al. (2010) evaluated two satellite products (CMORPH and 3B42-V6) over the Oklahoma region in the midwestern United States. The study pointed out that CMORPH tended to overestimate the precipitation volume more prominently than 3B42-V6 during the warm season while the bias of both satellite products was lower than $20 \%$ during the cold season. Yong et al. (2010) highlighted the geography-dependent (latitude and elevation) roles of the TMPA 3B42 product in near-real time (3B42-RT) and 3B42-V6 over the Laohahe basin in northeastern China. Meanwhile, better agreement with gauge observations was found for 3B42-V6 at both daily and monthly scales. Su et al. (2008) focused on the performance of 3B42-V6 over the La Plata basin in the Amazon. They concluded that the satellite estimates are slightly higher than the gridded gauge data at both monthly and daily time scales, with a higher degree of agreement for the monthly time scales. Other satellite error studies are those of Ali et al. (2005) and Dinku et al. (2007), who investigated the error structures of four satellite products over the Sahara and nine satellite products over the complex terrain of East Africa, respectively. They showed that the products are consistent with rainfall estimated from the ground-based gauge networks at a coarse resolution (i.e., $2.5^{\circ} \times 2.5^{\circ}$ grid cell at monthly interval).

Although the topic of satellite rainfall error analysis has been investigated globally for more than two decades (Anagnostou et al. 2010; Anagnostou 2004; Petty and Krajewski 1996; Arkin and Ardanuy 1989), only a small portion of these studies have focused on the error structure of satellite products on the event basis (Nikolopoulos et al. 2013; Mishra 2012; AghaKouchak et al. 2010); even fewer of them have attempted to decipher the error structure based on a large number of storm events (Stampoulis and Anagnostou 2012). Furthermore, the majority of the literature on this topic is generally focused on the error analysis at the satellite products' spatial (typically ranging between $0.04^{\circ}$ and $0.5^{\circ}$ ) and temporal scales (ranging between $15 \mathrm{~min}$ and daily). While using a regular space-time scale has allowed consistency in the evaluation of results from different error studies, it does not allow a direct interpretation of the satellite rainfall error in terms of hydrologic applications. In hydrologic modeling, particularly the basin flood response to precipitation, spatial and temporal scales are dictated by the basin's drainage area and duration of storm causing the flood event. Therefore, the current literature lacks comprehensive hydrologically driven satellite rainfall error studies that depict the error structure of basin-averaged satellite rainfall on the basis of long-term records of storm 
events. Understanding and improving satellite rainfall error characteristics at the basin scale, and on an event basis, will improve uses of satellite rainfall data in regional water budget analyses and for monitoring or forecasting of hydrologic extremes (flash floods and droughts).

This study attempts to evaluate the error characteristics of four quasi-global satellite precipitation algorithms (see section $2 \mathrm{~b}$ for the descriptions) over a mountainous area in the eastern Italian Alps. The surface rainfall data are derived from a dense rain gauge network over the study area. This error analysis is expected to give supplementary information and guidance to relevant studies regarding the uncertainties of satellite-derived precipitation estimates over complex mountainous regions and for heavy precipitation events. It is noted that, given the mountainous setting of the study domain, precipitation, particularly during cold months, could be in the form of snow or mixed phase, which is not discriminated in this study. In the next section, we describe the study area and data used. Section 3 introduces the error metrics and the score system. Results are reviewed in section 4, and conclusions are drawn in section 5.

\section{Study area and data}

\section{a. Study area}

This study focuses over the eastern part of the upper Adige River basin, a mountainous region located in the eastern Italian Alps (Fig. 1), particularly the Isarco basin $\left(4166 \mathrm{~km}^{2}\right)$ and the upper Passirio basin $\left(427 \mathrm{~km}^{2}\right)$. The basins have a mean elevation of $1736 \mathrm{~m}$ MSL with the highest (lowest) elevation at about 3700 (220) m MSL. The region is influenced by western Atlantic airflows and meridional circulation patterns (Frei and Schär 1998) causing HPEs and associated flash floods and debris flows in the summer and fall seasons. The dominant climate pattern in the region is continental, with the precipitation monthly distribution exhibiting two maxima, during August and October. The mean (maximum/minimum) annual precipitation accumulations in the 2003-10 period for the Isarco and Passirio basins were $651 \mathrm{~mm}$ (987/480) and $637 \mathrm{~mm}$ (865/482), respectively. The October-April period is typically dominated by snow and widespread type precipitation, while in the May-September period precipitation is mainly characterized by mesoscale convective systems and localized thunderstorms (Norbiato et al. 2009; Frei and Schär 1998).

\section{b. Rainfall data}

The study area is covered by a dense rain gauge network (87 gauges) with densities of contributing gauges per basin ranging between one station per $16 \mathrm{~km}^{2}$ (for the various analyzed subbasins) and $53 \mathrm{~km}^{2}$ (average gauge density for the entire area). The gauge rainfall record is hourly with an 8 -yr temporal coverage span (2003-10). Hourly gauge precipitation time series averaged over the study basins were generated using the nearest neighbor interpolation technique.

Four near-global satellite products are used in this study. The 3B42-RT product, which is corrected by monthly climatological gauge rainfall, and available in post-processing (version 7; hereafter named 3B42-V7) using current month gauge adjustments (Huffman et al. 2007), are from the National Aeronautics and Space Administration (NASA). Another IR-based precipitation product is the Precipitation Estimation from Remotely Sensed Information Using Artificial Neural Networks (PERSIANN), which uses a coincident MW calibrated neural network technique to relate IR observations to rainfall estimates (Sorooshian et al. 2000). The fourth product is the National Oceanic and Atmospheric Administration (NOAA) CMORPH, which uses multisatellite-based MW rain estimates integrated in space and time using motion vectors derived from IR images (Joyce et al. 2004). The spatial and temporal resolutions of the satellite rainfall products used in this study are $0.25^{\circ}$ at 3-hourly time intervals covering the same period as the gauge rainfall product. As with the rain gauges, all four satellite products were spatially interpolated to derive basin-averaged precipitation, using the nearest neighbor approach (taking the center of satellite pixel as the equivalent station location).

Since satellite products represent 3-hourly rainfall values, hourly rain gauge basin-averaged rainfall time series were averaged every three consecutive time steps (i.e., 0000, 0300, 0600, etc., UTC) so as to match the CMORPH and PERSIANN products. Since the two 3B42 products represent MW or IR rainfall estimates within $\pm 1.5 \mathrm{~h}$ of the synoptic hours, we have taken a different approach of matching gauges to this product. Namely, hourly basin-averaged rain gauge rainfall values were temporally averaged within $\pm 2 \mathrm{~h}$ around each synoptic hour to represent the 3-hourly temporal intervals of the $3 \mathrm{~B} 42$ product.

\section{Methodology}

A large number of precipitation events (3249) based on the 8-yr (2003-10) rain gauge record was grouped into warm (May-August) and cold (September-December) season months (the terms warm season months and MayAugust, as well as cold season months and SeptemberDecember, are used interchangeably in the text). These events were used to evaluate systematic and random error metrics of the three satellite products and their 


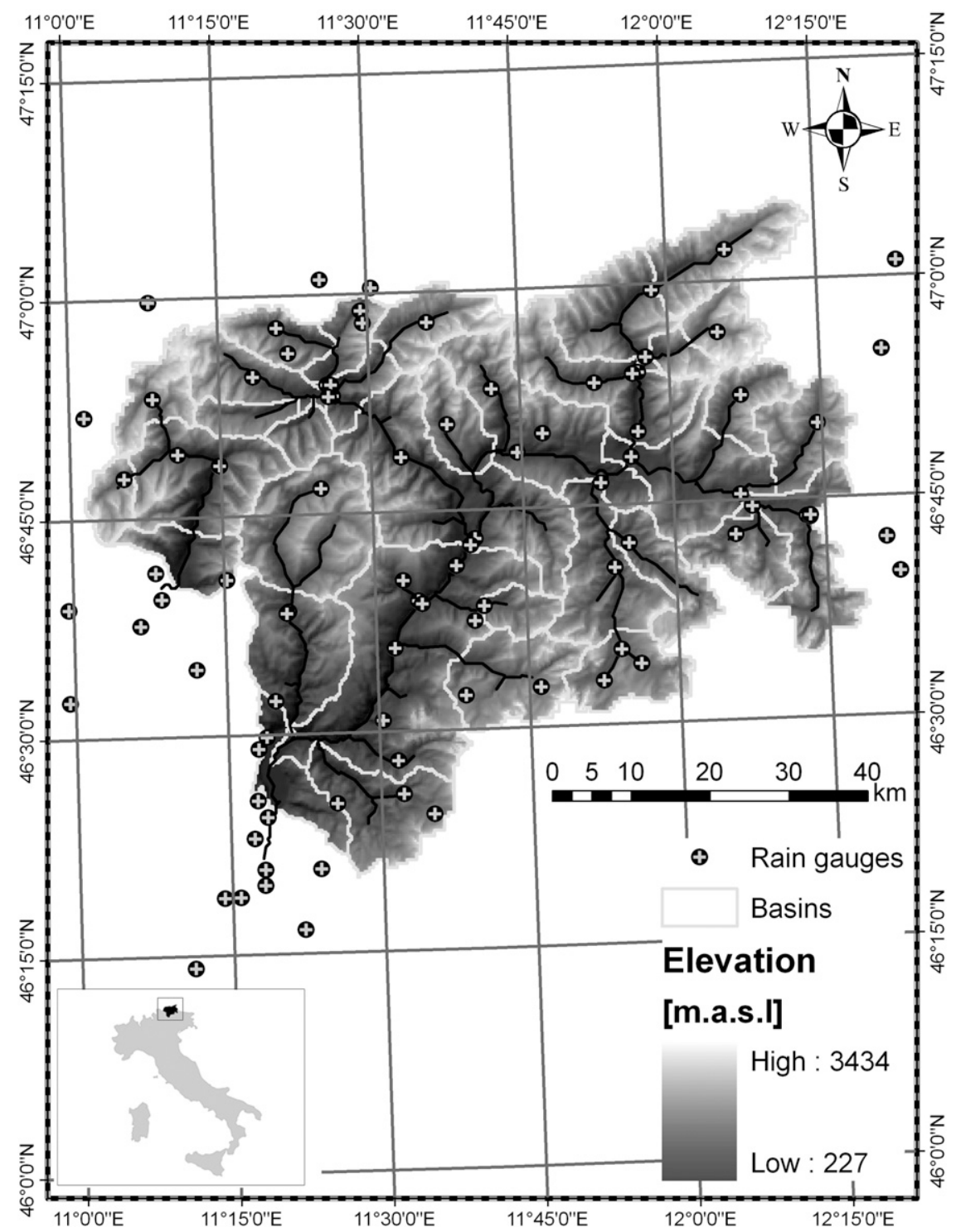

FIG. 1. Elevation map of the study area (eastern part of upper Adige River basin) and locations of available rain gauges. Inset map shows the location of study area over Italy, and the overlaid grid corresponds to the satellite grid $\left(0.25^{\circ} \times 0.25^{\circ}\right)$. The figure also shows boundaries of the subbasins used in the study.

dependency on seasonal characteristics of storms, basin scale, and event severity. Analysis was based on basinaveraged precipitation rather than the usual pixel-based comparison. This approach allows us a more direct inference on the hydrological impact of the satellite precipitation estimation error. In this study, thirteen basins with an area greater than $200 \mathrm{~km}^{2}$ were considered and summarized in Table 1. Basins with an area less than $530 \mathrm{~km}^{2}$ (namely, the approximate mean area of satellite pixels over the study area) are classified here as smallsized basins, while basins with area greater than this threshold are classified as medium sized.

\section{a. Event identification and matching}

Basin precipitation events were extracted from the rain gauge and satellite rainfall records for two distinct periods (May-August and September-December) using an ad hoc 9-h zero-rainfall time window to represent interstorm periods. The sensitivity of the results on the selected time window was investigated (not shown here) and found to be low for values in the range of $9-16 \mathrm{~h}$. Our choice to use the lower value was to allow the capture of the shorter-duration storms in our database. Precipitation events identified on the basis of the rain 
TABLE 1. Summary of basin information. S and M stand for small and medium.

\begin{tabular}{|c|c|c|c|c|c|}
\hline \multirow[b]{2}{*}{ Scale class } & \multirow[b]{2}{*}{ Area $\left(\mathrm{km}^{2}\right)$} & \multicolumn{2}{|c|}{$\begin{array}{c}\text { Elev } \\
(\mathrm{m} \mathrm{MSL})\end{array}$} & \multicolumn{2}{|c|}{ Gauges } \\
\hline & & Mean & STD & Number & Mean elev (m) \\
\hline S1 & 208 & 2040 & 606 & 13 & 1163 \\
\hline $\mathrm{S} 2$ & 236 & 1859 & 401 & 9 & 1076 \\
\hline S3 & 255 & 1894 & 448 & 12 & 1304 \\
\hline S4 & 345 & 1884 & 691 & 13 & 1388 \\
\hline S5 & 391 & 1892 & 516 & 10 & 1268 \\
\hline S6 & 417 & 1598 & 557 & 13 & 935 \\
\hline S7 & 427 & 1770 & 744 & 14 & 1258 \\
\hline S8 & 505 & 2008 & 618 & 16 & 1308 \\
\hline M1 & 1262 & 1979 & 673 & 26 & 1206 \\
\hline M2 & 1906 & 1958 & 687 & 44 & 1255 \\
\hline M3 & 1992 & 1951 & 690 & 46 & 1231 \\
\hline M4 & 2863 & 1904 & 758 & 68 & 1230 \\
\hline M5 & 4166 & 1770 & 838 & 85 & 1185 \\
\hline
\end{tabular}

gauge (i.e., reference) and satellite basin-averaged precipitation time series were matched according to their centroid differences as follows:

$$
\left|t_{c, s}-t_{c, g}\right| \leq R
$$

where $t_{c, s}$ and $t_{c, g}$ are the centroids of the satellite and gauge precipitation events defined as

$$
t_{c}=\frac{\sum_{t=1}^{T_{s}} t[p(t)]}{\sum_{t=1}^{T_{s}} p(t)},
$$

where $p(t)$ is the basin-averaged precipitation rate $\left(\mathrm{mm} \mathrm{h}^{-1}\right)$ at each time step $t(3 \mathrm{~h})$ of the event duration $T_{S}$. The variable $R$ is defined based on the reference data as

$$
R=\max \left(t_{c}-t_{b}, t_{e}-t_{c}\right),
$$

where $t_{b}$ and $t_{e}$ are the beginning and ending time of the gauge-defined precipitation events, respectively. It is possible that for a given gauge-defined precipitation event with $t_{c, g}$ there is more than one eligible satellitedefined precipitation event. In those cases, the satellite precipitation events were merged into one event. Precipitation events with cumulative basin-averaged reference precipitation greater than $3 \mathrm{~mm}$ were considered in this study to eliminate minor events with negligible hydrologic response.

Table 2 lists the properties of the selected events. As noted from the table, the May-August period has

\begin{tabular}{|c|c|c|c|c|c|c|}
\hline \multirow[b]{2}{*}{ Scale class } & \multicolumn{2}{|c|}{ No. of events } & \multicolumn{2}{|c|}{ Duration (h) } & \multicolumn{2}{|c|}{$\begin{array}{c}\text { Rainfall } \\
\text { accumulation } \\
(\mathrm{mm})\end{array}$} \\
\hline & Warm & Cold & Warm & Cold & Warm & Cold \\
\hline S1 & 181 & 90 & 36 & 52 & 17 & 25 \\
\hline S2 & 153 & 80 & 46 & 59 & 15 & 20 \\
\hline S3 & 161 & 98 & 58 & 56 & 18 & 19 \\
\hline S4 & 180 & 92 & 41 & 58 & 15 & 22 \\
\hline S5 & 199 & 107 & 44 & 49 & 19 & 21 \\
\hline S6 & 117 & 75 & 53 & 61 & 16 & 18 \\
\hline S7 & 170 & 86 & 42 & 61 & 14 & 20 \\
\hline S8 & 156 & 87 & 66 & 72 & 21 & 26 \\
\hline M1 & 159 & 90 & 67 & 67 & 18 & 18 \\
\hline M2 & 157 & 93 & 74 & 71 & 20 & 19 \\
\hline M3 & 156 & 92 & 74 & 71 & 20 & 19 \\
\hline M4 & 153 & 88 & 82 & 84 & 21 & 21 \\
\hline M5 & 147 & 82 & 86 & 90 & 18 & 20 \\
\hline
\end{tabular}
a larger number of precipitation events (nearly twice as
TABLE 2. Summary of event properties.

much as the September-December period), but events in the September-December period have longer durations and higher rainfall accumulations due to the distinct meteorological patterns in these two periods. It is also noted that the basin-averaged precipitation accumulations $R_{V}$ for the May-August period events are lower than those of the September-December period events for the small-scale basins, while for the medium-scale basins the $R_{V}$ values tend to be similar in the two periods. Figure 2 shows the empirical cumulative density functions (CDFs) of the precipitation event durations, basin-averaged precipitation accumulation, and maximum event precipitation rates derived from the reference data. As shown in Table 2, small basin-scale precipitation events in the September-December period exhibit longer durations when compared to the May-August period events, and this parallels with higher rainfall accumulations during the SeptemberDecember period relative to the May-August period. It is also noted that in the September-December period, small-sized basin events have larger population in low quantiles $\left(<12 \mathrm{~mm} R_{V}\right)$, yet the population in high quantiles is smaller relative to the $R_{V}$ values from the medium-sized basin-scale events. Moreover, it is noted that small-basin warm season precipitation accumulation CDFs exhibit lower precipitation accumulations than those in cold season months, while the distributions of maximum precipitation rates for events in both medium and small basins during warm season months exhibit slightly higher values than those in cold months.

The precipitation events were grouped according to values of basin-averaged precipitation accumulation associated with the 50th, 80th, 90th, and 95th percentiles. Values below the 50th percentile were associated 

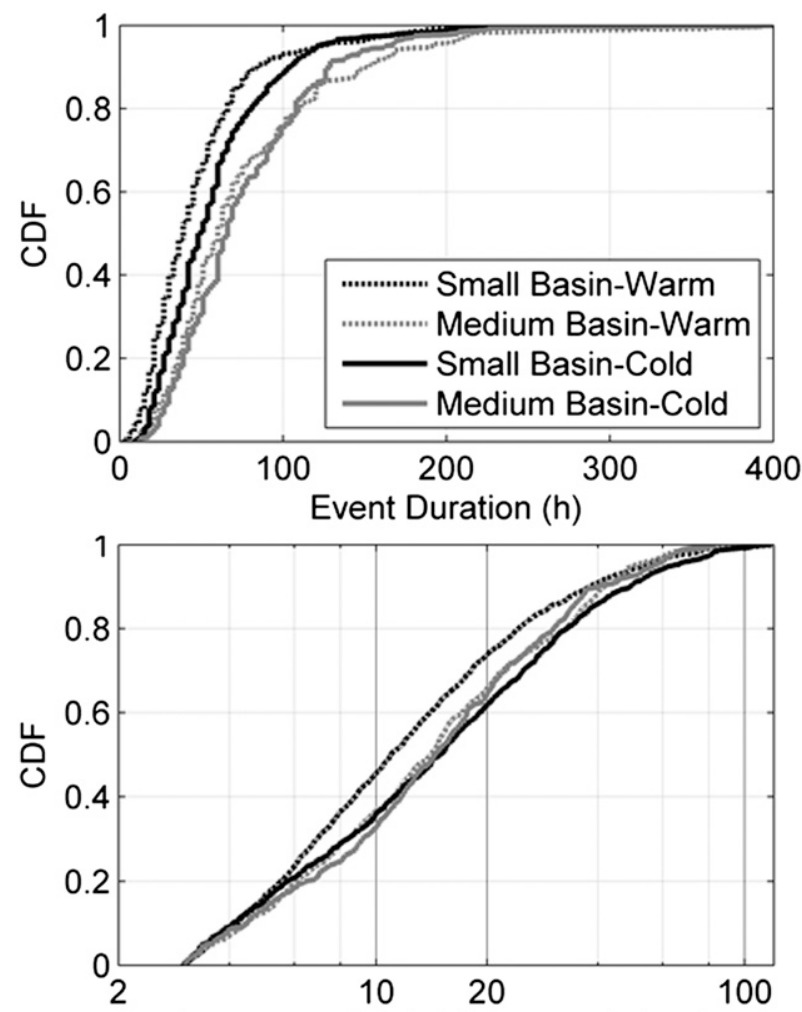

Basin-averaged Rainfall Accumulation ( $\mathrm{mm}$ )

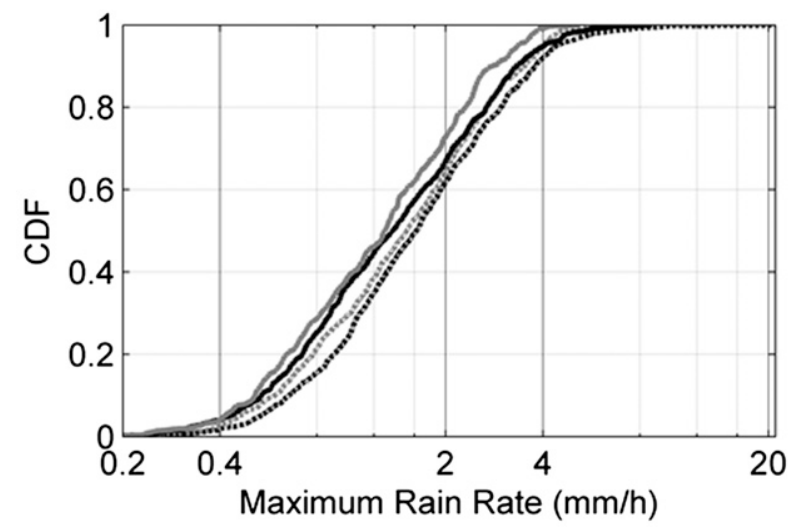

FIG. 2. Event-based empirical CDFs of (top) event duration $D$, (middle) basin-averaged rainfall accumulation $R_{V}$, and (bottom) max rain rate $R_{M}$; all derived from reference rainfall.

with low rainfall accumulation $(<10 \mathrm{~mm}$; see Table 3$)$ and are excluded from this analysis since our interest is toward moderate to heavy precipitation events. The quantile values for the different basin scales and periods are summarized in Table 3. It is noted that the quantile values are greater in the September-December months than those for the May-August months, which is consistent with the cumulative distributions shown in Fig. 2 and points to the contrasting precipitation properties in these two periods.

\section{b. Evaluating metrics}

Three evaluation metrics termed as relative centroid displacement $d_{c}$, multiplicative error $\varepsilon$, and a herein established accuracy index AI are selected to describe the degree of disagreement between reference (i.e., gauge precipitation) data and the four satellite-derived precipitation products.

A number of studies have shown that temporal error characteristics in precipitation estimates may propagate to the simulated hydrograph-producing timing errors (Mei et al. 2014; Nikolopoulos et al. 2013; Zoccatelli et al. 2011; Yong et al. 2010; Su et al. 2008; Sharif et al. 2002). However, to the best of our knowledge, these error characteristics have not been exploited to develop a metric for the evaluation of satellite precipitation estimates. The relative centroid displacement, Eq. (4), is therefore proposed herein as a metric to depict the error in estimating from satellite observations the time of arrival of the event temporal center of mass:

$$
d_{c}=\frac{t_{c, s}-t_{c, g}}{D_{g}},
$$

where $D_{g}$ stands for the duration of gauge precipitation event defined in Eq. (6). The numerator in Eq. (4) represents the centroid displacement. Since we have shown that the durations of events in September-December are generally shorter than those in May-August, we normalized the net centroid displacements to the corresponding gauge-derived event durations. By the definition of $t_{c}$ from Eq. (2), $t_{c, s} / t_{c, g}$ represents the temporal centroid location in terms of satellite- or gauge-retrieved basin-averaged precipitation rate for matching event pairs; thus, positive and negative $d_{c}$ values represent delay and advance in arrivals of storm center of mass, correspondingly. In practice, $d_{c}$ reflects the situation of either early or delayed detection of storm events, which could be an important property when dealing with prediction of a basin's hydrologic response.

The multiplicative error $\varepsilon$, defined as the ratio between gauge-event properties to the corresponding satellite-event property, is one of the classical error metrics used in satellite precipitation error studies (e.g., Hossain and Anagnostou 2006):

$$
\varepsilon=\frac{I_{S}}{I_{G}},
$$

where $I_{S}$ and $I_{G}$ are the basin-averaged rainfall properties derived from satellite products and gauges. The event properties are defined as follows:

$$
\text { event duration : } D=t_{e}-t_{b} \text {; }
$$


TABLE 3. Threshold values of basin-averaged precipitation accumulation (mm) associated with the 50th, 80th, 90th, and 95th quantiles.

\begin{tabular}{|c|c|c|c|c|c|c|c|c|}
\hline \multirow[b]{2}{*}{ Scale class } & \multicolumn{2}{|c|}{ Q50 } & \multicolumn{2}{|c|}{ Q80 } & \multicolumn{2}{|c|}{ Q90 } & \multicolumn{2}{|c|}{ Q95 } \\
\hline & Warm & Cold & Warm & Cold & Warm & Cold & Warm & Cold \\
\hline S1 & 11 & 14 & 22 & 38 & 31 & 53 & 44 & 62 \\
\hline S2 & 9 & 13 & 19 & 33 & 33 & 43 & 37 & 54 \\
\hline S3 & 8 & 15 & 20 & 28 & 38 & 36 & 49 & 49 \\
\hline S4 & 8 & 13 & 23 & 23 & 42 & 39 & 59 & 46 \\
\hline S5 & 13 & 14 & 29 & 28 & 39 & 40 & 48 & 61 \\
\hline S6 & 14 & 16 & 33 & 32 & 45 & 44 & 58 & 63 \\
\hline S7 & 12 & 17 & 24 & 39 & 33 & 60 & 57 & 77 \\
\hline S8 & 14 & 16 & 30 & 38 & 52 & 63 & 56 & 79 \\
\hline M1 & 12 & 14 & 27 & 28 & 37 & 37 & 48 & 55 \\
\hline M2 & 13 & 13 & 30 & 27 & 37 & 37 & 42 & 47 \\
\hline M3 & 13 & 16 & 28 & 32 & 40 & 45 & 51 & 59 \\
\hline M4 & 14 & 14 & 33 & 30 & 43 & 37 & 50 & 52 \\
\hline M5 & 15 & 14 & 34 & 31 & 43 & 37 & 50 & 53 \\
\hline
\end{tabular}

event rainfall accumulation : $R_{V}=\sum_{t=1}^{T_{s}} p(t) ; \quad$ and

event maximum rainfall rate $: R_{M}=\max _{t \in T_{s}}[p(t)]$

Values of $\varepsilon$ greater or smaller than one correspond to overestimation or underestimation, respectively, of the satellite product related to the reference for a given event property.

We introduce in this study an error metric (AI) that is based on the ratio of the geometric to the arithmetic means of the satellite product and gauge-based event properties $\left(I_{S}\right.$ and $\left.I_{G}\right)$ :

$$
\mathrm{AI}=\frac{2 \sqrt{I_{S} I_{G}}}{I_{S}+I_{G}} .
$$

The variable $\mathrm{AI}$ is bounded between 0 and 1 , given that the geometric mean would be always less than or equal to the arithmetic mean (Steele 2004). The variable AI is more comprehensive than $\varepsilon$ in that it incorporates both magnitude and rain detection discrepancies in the satellitederived products. Specifically, when either $I_{S}$ or $I_{G}$ is 0 , AI would equal 0 , denoting either missing or a false alarm of satellite estimate; on the other hand, if $I_{S}$ is equal to $I_{G}$, AI is 1, which implies a perfect match between the two datasets. We attempt to visualize this index by relating it to the multiplicative error metric $\varepsilon$ as follows:

$$
\mathrm{AI}=\frac{2 \sqrt{\varepsilon}}{\varepsilon+1}
$$

Figure 3 (top) shows the above relationship. As noted, AI is symmetric (in $\log$ scale) with respect to $\varepsilon=1$ (unbiased estimator), which divides the error into underestimation (left side) and overestimation (right side). As an example, if $\varepsilon$ equals $0.2,0.1$, or $0.02(5,10$, and 50$)$, the corresponding AI values are $0.75,0.57$, or 0.28 , respectively, which indicates a nonlinear relationship between the two metrics.

The variables $\mathrm{AI}$ and $\varepsilon$ will be used in this analysis to provide complementary evaluations of the different satellite products. Specifically, $\varepsilon$ will define the degree of overestimation (underestimation) of the satellite products for each event property separately, while AI will enable a combined evaluation of the three event properties $\left(D, R_{V}\right.$, and $\left.R_{M}\right)$ integrated into a score system defined by a triangle with area $A$ determined as

$$
A=\frac{\sin \alpha}{2} \mathrm{AI}_{D} \mathrm{AI}_{V}+\frac{\sin \alpha}{2} \mathrm{AI}_{V} \mathrm{AI}_{M}+\frac{\sin \alpha}{2} \mathrm{AI}_{M} \mathrm{AI}_{D},
$$

where $\mathrm{AI}_{D}, \mathrm{AI}_{V}$, and $\mathrm{AI}_{M}$ represent the $\mathrm{AI}$ values for $D$, $R_{V}$, and $R_{M}$, and $\alpha$ (equal to $120^{\circ}$ ) is the angle between the different axes (Fig. 3, bottom). This triangle area is normalized with respect to the maximum triangle area to derive a score index $(S)$ ranging between 0 (missing or false alarm) and 1 (accurate matching):

$$
S=\frac{\mathrm{AI}_{D} \mathrm{AI}_{V}+\mathrm{AI}_{V} \mathrm{AI}_{M}+\mathrm{AI}_{M} \mathrm{AI}_{D}}{3} .
$$

The variable $S$ is employed in this study as an indicator of satellite skill determined for each event separately or on the basis of all events combined. One of the main assumptions of our formulation is that the three event properties are considered having equal weight in evaluating the score index for the satellite rainfall products. The weighting of the different event properties in the 

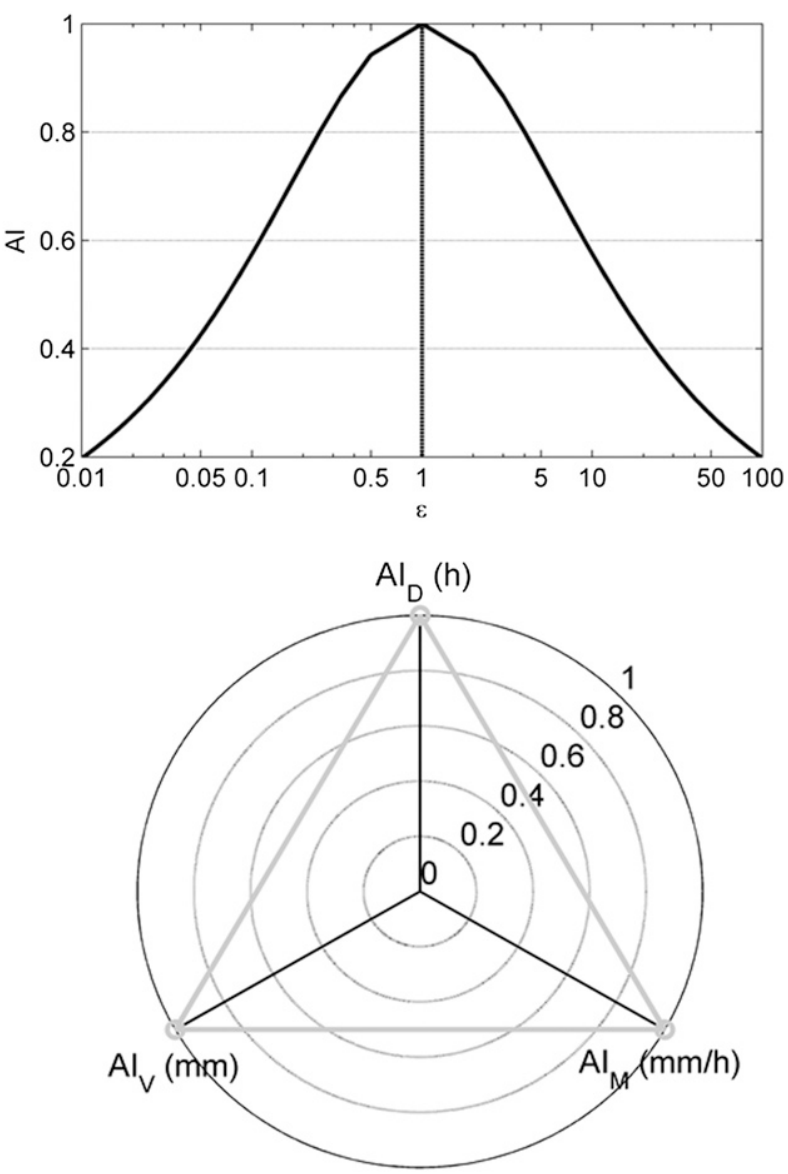

FIG. 3. (top) The relationship between AI and $\varepsilon$. (bottom) Schematic depicting the way $S$ is calculated.

score index is an aspect that could be evaluated in future research on the basis of the relative significance of these properties on the flood hydrograph.

\section{Results}

\section{a. Displacement of centroid}

As discussed in section $3 \mathrm{~b}$, the displacement of a basin-averaged precipitation centroid is an important error property that relates to the application of satellite precipitation data for simulating basin flood response. In Fig. 4, we show the displacement between event centroid locations defined in Eq. (4). Results suggest that the displacement in the event centroid is random, implying no preference on either advance or delay of detection, because values of $d_{c}$ display neither scale nor quantile dependency and distribute around zero (refer to the median location). However, as quantile range increases in value, the range of $d_{c}$ becomes narrower in most of the cases, pointing to the fact that satellite precipitation events from high quantile ranges have better consistency in terms of matching the timing of a storm's centroid. Apparently, high-quantile events are associated with long duration, which results overall in a reduced relative displacement. It could thus be stated that the effect introduced by the shift in centroid is more significant for shorter-duration events. Besides, events from medium basin scales tend to have smaller $d_{c}$ value ranges, again due to the longer event duration of this event class, which reduces the $d_{c}$. A product-wide comparison indicates that none of the products has distinctly good or bad performance by means of arrival of the centroids compared to the others, but the two $3 \mathrm{~B} 42$ products show better convergence trend as quantile ranges increase. Consequently, the timing error being propagated to the hydrograph is more pronounced for shorter-duration events (events typically from small basin and convective rainfall system).

\section{b. Multiplicative error analysis}

Figure 5 shows the box plots of the event-based multiplicative error $(\varepsilon)$ for different satellite products, that is, quantile ranges, and the two basin scales over the May-August and September-December months. A first observation from Fig. 5 is that the satellite estimates, especially CMORPH and PERSIANN, during the September-December period tend to underestimate the gauge rainfall in all quantile ranges and at both basin scales. These results are in general agreement with other studies that have shown significant underestimation from CMORPH and PERSIANN techniques over complex terrain and during cold season months due to snow contamination and low-level orographic enhancement (AghaKouchak et al. 2011; Tian et al. 2009; Dinku et al. 2007). On the other hand, the two 3B42 products (V7 and RT) exhibit better consistency with the gauge-based reference data in both periods and basin scales. A reason behind this could be that the two $3 \mathrm{~B} 42$ products are adjusted by monthly gauge datasets. While the gauges that TMPA uses in the monthly adjustment are not the same as the gauge data in the analysis, it is expected that a certain amount of correlation between the two gauge datasets exists and influences the statistics.

Furthermore, from these results a magnitude-dependent error structure is noted, ranging from overestimation to underestimation as the quantile range increases. In addition, results for the $3 \mathrm{~B} 42$ products indicate a convergence trend (namely, shorter 25th-75th interquartile range, shorter 5th-95th percentile ranges, and decreasing number of outliers) in the $\varepsilon$ values toward the higher quantiles of reference precipitation accumulation and for the medium-sized basins. This trend is not apparent for the other two products (only PERSIANN during cold 

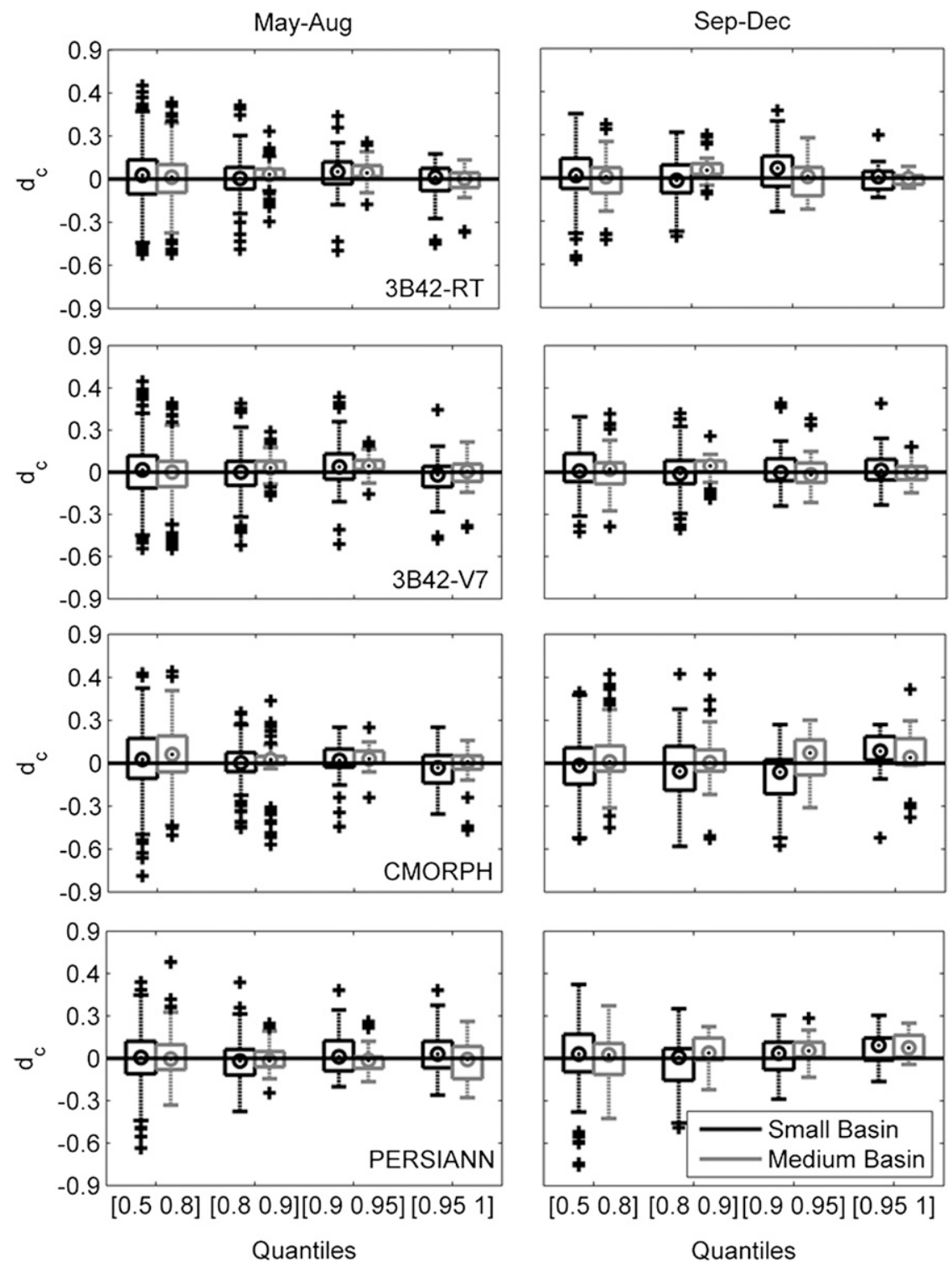

FIG. 4. Box plots of event centroid displacement between the various satellite products and gauge-derived basin-averaged rainfall: (left) May-August and (right) September-December.

months is showing a convergence and for only the medium-sized basins). It is also noted from the box plots that the median of $\varepsilon$ values for the small-scale basins is more skewed relative to the medium-sized basins for CMORPH and PERSIANN, pointing to the fact that the satellite precipitation error for the medium-sized basins is better represented by the mean value. It is briefly summarized here that satellite precipitation estimates for smaller basins, higher quantile ranges, or months with colder temperatures have lower values of multiplicative error (namely, satellite underestimation), while for lower quantile ranges and larger-sized basins they exhibit overestimation.
A more focused analysis on the most severe events (precipitation accumulations greater than the 90th quantile) was conducted, with results reported in Fig. 6 scatterplots and corresponding statistics reported in Table 4. Significant scatter is noted in all comparisons of satellite versus reference basin-averaged precipitation accumulations. It is noted that underestimation is the most dominant scenario, particularly for CMORPH and PERSIANN products during cold months, which matches the multiplicative error distributions shown in Fig. 5. Visually, 3B42-V7 has better correlation to gauge-based basin-averaged precipitation accumulation, which is also statistically supported by the correlation 

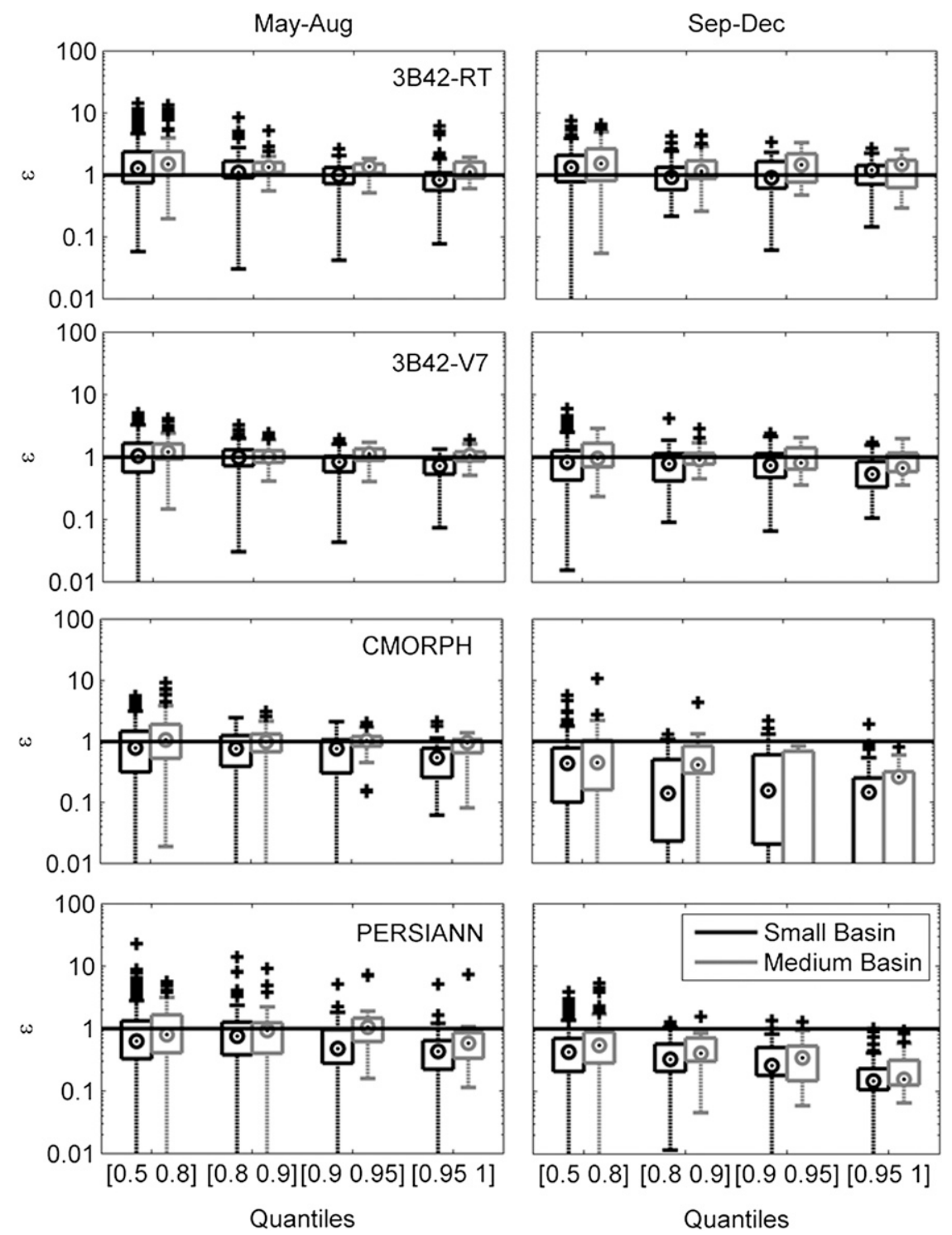

FIG. 5. As in Fig. 4, but for event multiplicative error between the various satellite products and gauge-derived basin-averaged rainfall.

coefficients (CCs) reported in Table 4 (CC for the 3B42V7 warm period is 0.51 , the highest). This is expected since $3 \mathrm{~B} 42-\mathrm{V} 7$ is adjusted to the actual monthly gauge. However, 3B42-V7 is ambiguously correlated to gauge during September-December months even with its gauge-adjusted feature. A possible explanation of this could again be the snow contamination during the cold period of the study region. Surprisingly, the cold-period scatterplot for 3B42-RT exhibits better linearity compared to its post-real-time counterpart in Fig. 6, with the highest $C C$ value (0.34) and relatively higher (but in absolute terms low) $\mathrm{CC}$ value in the May-August period (0.38), displayed in Table 4. It seems that during the cold period the monthly climatological gauge-adjusted feature from the real-time 3B42 has a higher degree of influence remaining in the event-based precipitation accumulation compared to the actual monthly gauge adjustments from the post-real-time 3B42. Besides CC, the root-mean-square error (RMSE) statistic is also rendered for the comparison purpose. It is seen that the RMSE values for 3B42-V7 are the lowest for both periods, indicating better consistency with the reference precipitation accumulation than the other two products. CMORPH and PERSIANN are marked by low correlation and high magnitude discrepancies. Finally, all satellite rainfall products have nearly null NashSutcliffe index values, meaning that these estimates perform merely as the mean of reference data in terms of 

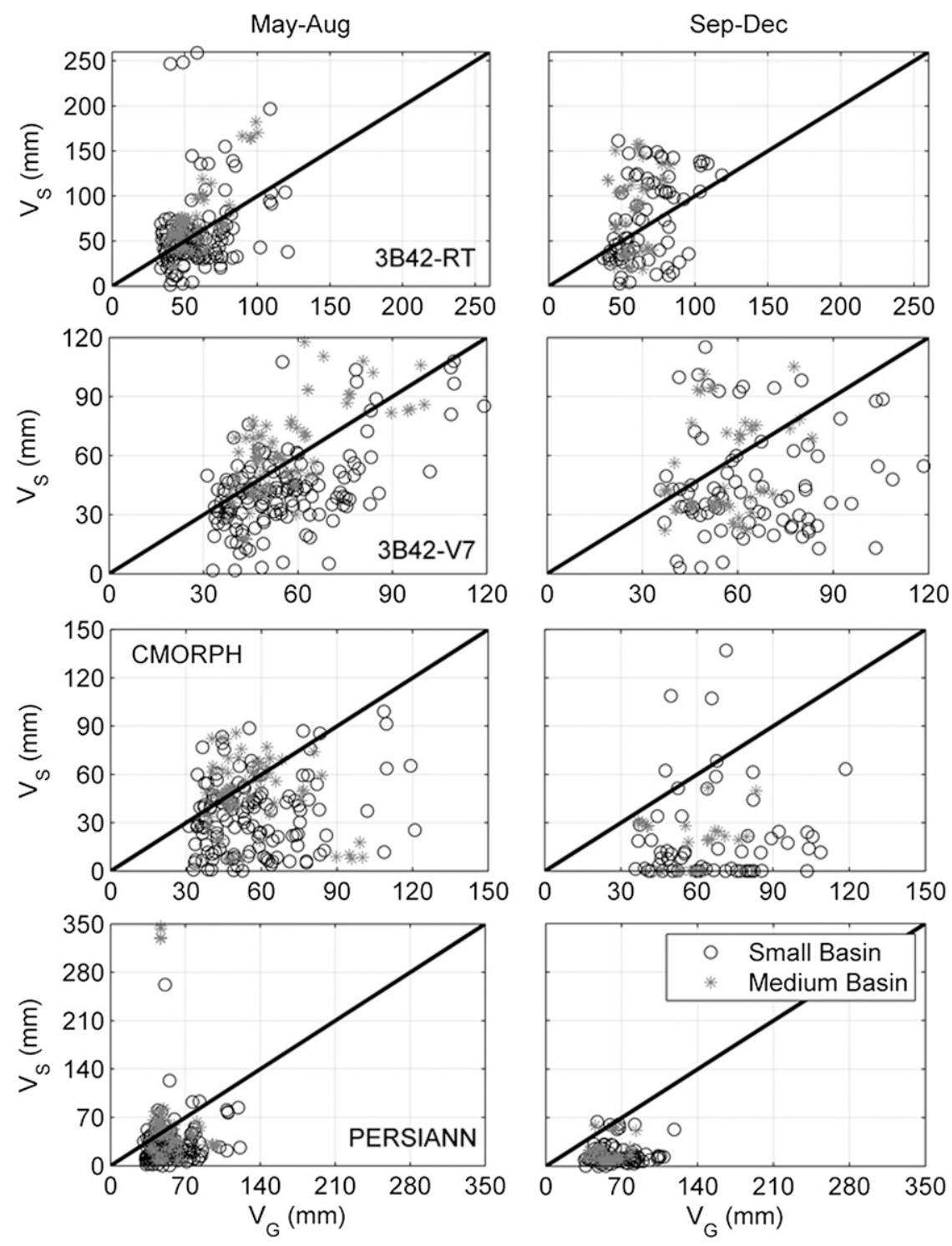

FIG. 6. Scatterplot of satellite product vs gauge-derived basin-averaged rainfall accumulation for events above Q90: (left) May-August and (right) September-December.

predicting small-to-medium basin-scale event rainfall accumulations.

\section{c. Score system analysis}

The score system, defined in section $3 b$, was applied on the four satellite products by averaging the AI values over events belonging in the Q80-Q100 quantile range for the two seasons and basin sizes, with results demonstrated in Fig. 7. The figure shows that AI values of duration for the products are all above 0.9 with negligible distinction (except for CMORPH, with identifiably weaker performance in predicting the event durations), implying reasonable and similar performances in capturing the duration of the reference precipitation. This is anticipated since the analyzed event population is for the most significant events (above the 80th quantile), which in general are associated with long durations and high rainfall accumulations; thus, the detection error by satellite is expected to be low. Although the rainfall duration is captured well, satellite estimates of basinaveraged storm total and maximum precipitation rate exhibit considerable uncertainty. As shown in Fig. 7, the $\mathrm{AI}_{V}$ and $\mathrm{AI}_{M}$ values are high for the May-August months (around 0.9) but considerably low for the September-December months, except for the two 3B42 products, which both have values still above 0.9 . This finding confirms the results from Fig. 5 that the error is quite close to 1 for the warm period but notably distant 
TABLE 4. Satellite product evaluation statistics for Q90 storm events. Boldface indicates best results among products.

\begin{tabular}{llcccc}
\hline \hline Periods & Statistics & 3B42-RT & 3B42-V7 & CMORPH & PERSIANN \\
\hline Warm & CC & 0.38 & $\mathbf{0 . 5 1}$ & 0.06 & 0.00 \\
& RMSE & 0.66 & $\mathbf{0 . 3 8}$ & 0.57 & 0.98 \\
Cold & CC & $\mathbf{0 . 3 4}$ & 0.04 & 0.11 & 0.06 \\
& RMSE & 0.72 & $\mathbf{0 . 5 4}$ & 0.86 & 0.80 \\
\hline
\end{tabular}

from 1 for cold months, particularly for the CMORPH and PERSIANN. It is specifically shown that AI for precipitation accumulation and maximum rain rate of CMOPRH are exceptionally low, ranging between 0.5 and 0.7 (0.7-0.9 for PERSIANN). Based on the evaluations on duration and magnitude in terms of AI, we can state that the snow contamination effect has a much stronger impact on the estimation of precipitation magnitude than that on duration in the event basis. Consequently, it appears that the CMORPH and PERSIANN algorithms lack the accuracy in those complex terrain heavy precipitation events, while $3 \mathrm{~B} 42$ products can provide a more accurate estimation of the three storm parameters (rainfall accumulation, maximum rainfall rate, and duration).

A consecutive investigation on the score $(S)$ for the storm events exceeding the 80th percentile is shown in Table 5 and Fig. 8. Table 5 lists the $S$ values from Fig. 7 (numbers in boldface represent the best estimates based on seasons and basin sizes). The two 3B42 products surpass the other two satellite products to a great extent, with $S$ being over 0.9 . The 3B42-RT scores no worse
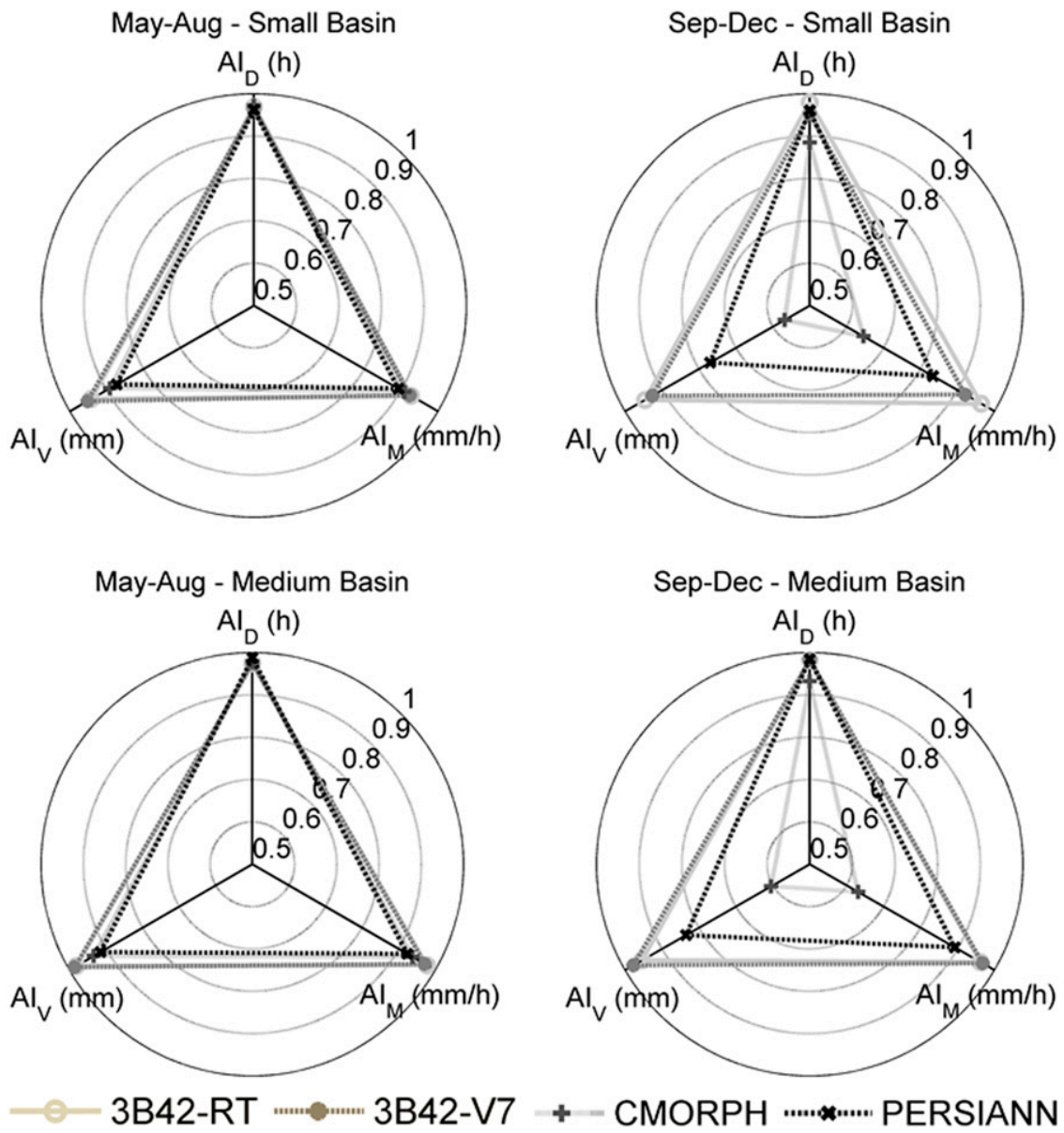

FIG. 7. Polar plots of AI for the three event properties determined for the different seasons and basin scales: (left) May-August and (right) September-December; (top) small and (bottom) medium basin. 
TABLE 5. Comparison of products scores for different periods and scales. Boldface indicates best results among products.

\begin{tabular}{|c|c|c|c|c|c|c|}
\hline Periods & Statistics & Scales & 3B42-RT & $3 \mathrm{~B} 42-\mathrm{V} 7$ & CMORPH & PERSIANN \\
\hline \multirow[t]{4}{*}{ Warm } & Mean & Small & 0.90 & 0.90 & 0.86 & 0.84 \\
\hline & & Medium & 0.96 & 0.95 & 0.91 & 0.89 \\
\hline & STD & Small & 0.14 & 0.13 & 0.17 & 0.18 \\
\hline & & Medium & 0.05 & 0.04 & 0.16 & 0.13 \\
\hline \multirow[t]{4}{*}{ Cold } & Mean & Small & 0.93 & 0.88 & 0.55 & 0.74 \\
\hline & & Medium & 0.93 & 0.95 & 0.58 & 0.82 \\
\hline & STD & Small & 0.09 & 0.13 & 0.35 & 0.21 \\
\hline & & Medium & 0.06 & 0.05 & 0.39 & 0.16 \\
\hline
\end{tabular}

than 3B42-V7 with a few obscure differences. Meanwhile, the values of standard deviation (STD) of $S$ for 3B42-V7 are the smallest, except for the small-scale cold-period case. It can be inferred that smaller STDs could indicate better performance given the median of $S$ is close to 1, namely, $S$ values tend to locate toward 1 . The box plots of $S$ determined for each product for the two seasons and basin scales are juxtaposed in Fig. 8 . The results reveal our findings according to Fig. 7. Again 3B42-V7 and RT show better consistency to gauge data with median locations fairly close to unity and low variability of $S$. In addition, the median locations for the other two products are apart from 1 with considerable large variability in $S$ values (no outliers for CMORPH and PERSIANN in cold season), demonstrating worse performances compared to the two 3B42 products. In terms of seasonality, May-August months, exhibit a smaller quantile range compared to the SeptemberDecember months. To summarize, 3B42-V7 could be an eligible algorithm in retrieving rainfall over warm season months while its unadjusted version (3B42-RT) provides sensible precipitation estimates in the cold season months. Overall, both 3B42 precipitation products are shown to outperform CMORPH and PERSIANN algorithms in terms of the $S$ score examined; the algorithms of CMORPH and PERSIANN should be improved for cold season precipitation.

\section{Conclusions}

In this study, we review and evaluate the performance of four widely used global-scale satellite products over a mountainous area using variable spatiotemporal scales for comparison. Specifically, satellite products are evaluated
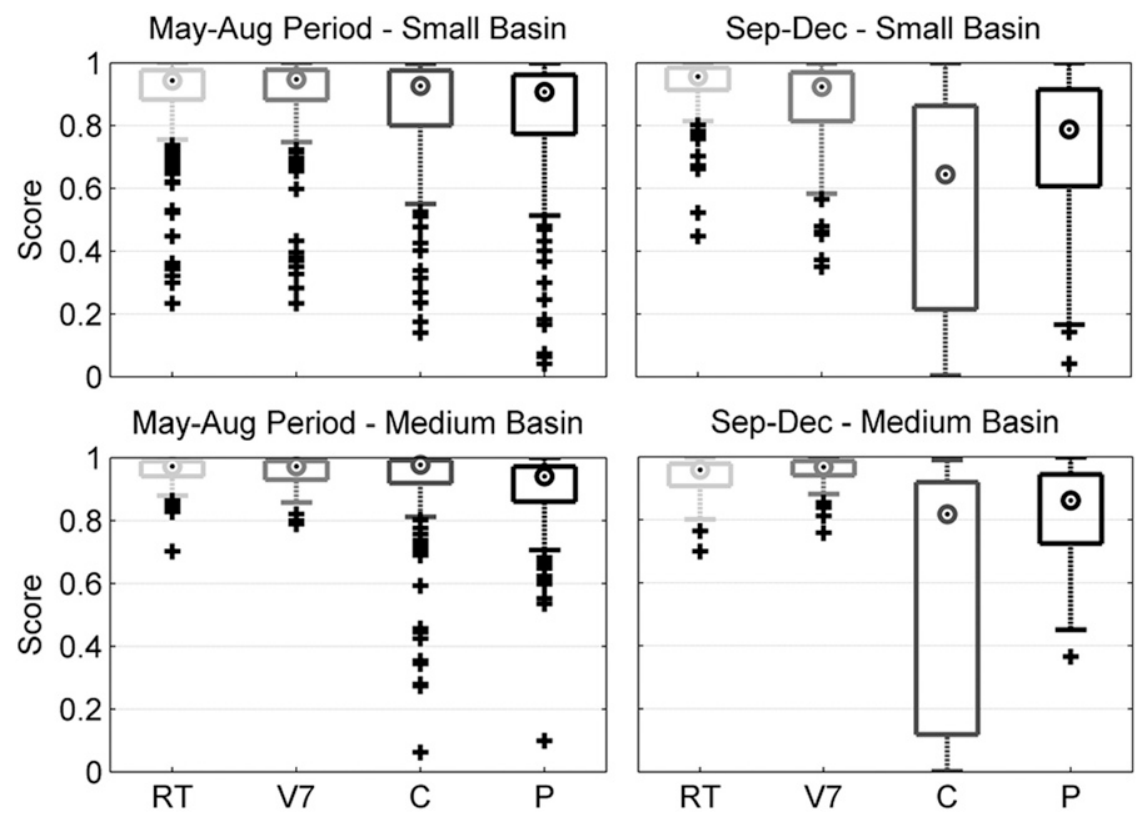

FIG. 8. Box plots of score for the four satellite rainfall products based on events above Q80 (RT, V7, C, and P stand for 3B42-RT, 3B42-V7, CMORPH, and PERSIANN) : (left) MayAugust and (right) September-December; (top) small and (bottom) medium basin. 
for separate storm events and different basin areas. Three evaluating metrics, namely, relative centroid displacement $\left(d_{c}\right)$, multiplicative error $(\varepsilon)$, and a score index $(S)$, are used to quantify the satellite precipitation estimation performance. The $d_{c}$ is a metric for depicting the timing error of the precipitation event; $\varepsilon$ represents the multiplicative error (i.e., bias ratio) in the event basin-averaged precipitation accumulation; and $S$ accounts for the errors in three different properties of the storm events: the event duration, basin-averaged precipitation accumulation, and event maximum rainfall rate. The variable $S$ is an error metric newly defined in this study, based on an accuracy index (AI) metric that was shown to be nonlinearly related to $\varepsilon$. Although AI cannot display the direction of error, it has the virtue of value unity (possible value space is bounded between 0 and 1), which allows a universal comparison of different precipitation, or hydrologic properties, in event scale.

It was shown that there is no clear trend in either the delay, or advance, in detection over different event precipitation accumulation quantiles, seasons (summer versus fall), or basin scales for the different satellite products. The variability of disagreement in the event-based basinaveraged precipitation centroid was shown to be more prominent for short-duration events over small-scale basins and low event-precipitation accumulations regardless of the satellite precipitation product. This implies that we cannot discriminate between satellite products in terms of timing error for hydrologic simulations.

On the other hand, all satellite products were shown to exhibit significant uncertainty in the estimation of basin-averaged precipitation accumulation at the event basis as indicated by $\varepsilon$. The degree of discrepancy is shown to vary between summer and fall months, basin scale, and event severity (surrogated by basin-averaged precipitation accumulation in this paper). A trend of overestimation to underestimation with increasing the quantile ranges of basin-averaged precipitation accumulation was shown and was particularly apparent for the CMORPH and PERSIANN products. The uncertainty of this trend, visualized as the value range of $\varepsilon$, generally decreases with increasing precipitation accumulation quantile values and basin scale (CMORPH in cold season was an exception). For heavy precipitation events, the results demonstrated that the two $3 \mathrm{~B} 42$ products exhibit better correlation as well as a lower degree of disagreement (quantified by RMSE) to the gauges, while CMORPH and PERSIANN significantly underestimated the reference data (particularly in the fall to early winter months period).

Similar results are established from the AI-based $S$ index. The predictive accuracy of satellite products for the selected event properties (event duration, basin-averaged storm total, and maximum precipitation rate) in heavy precipitation events during the summer months is acceptable ( $S$ greater than 0.9 ), especially in estimating the storm duration. A reasonable prediction (AI for duration above 0.9) for the duration is also shown during the fall to early winter months. However, the retrieved basin storm accumulation and maximum precipitation rates are inaccurate for CMORPH and PERSIANN for the September-December months. A slight decrease of the AI for basin-averaged precipitation accumulation and max rate is also observed for the 3B42-RT and 3B42-V7 products. Overall, the $S$ index for 3B42-V7 and its realtime version (3B42-RT) are concentrated near unity with a higher degree of centralization for the mediumsized basins during summer months. Product 3B42-V7 is shown to be the best product for predicting event precipitation associated with convective systems during summer months, while 3B42-RT outperformed 3B42$\mathrm{V} 7$ in the estimation of the cold-period precipitation events occurring over small-scale basins. The evaluation of cold-period precipitation events over medium-sized basins was equally satisfactory by the two $3 \mathrm{~B} 42$ products, while the usage of CMORPH or PERSIANN estimates exhibited low $S$ indices.

Although the study is based on a long data record (8yr), it represents a limited hydroclimatic and geomorphologic regime, and results can only be generalized for similar mountainous regions and orographic-driven precipitation events. Furthermore, given the mountainous setting and the early winter cold months considered in the study, we note the varying effects that snow-covered surfaces and mixed-phase precipitation can have on the satellite retrievals examined herein. Specifically, CMORPH is particularly prone to the effect of snow screening on MW rainfall estimates, typically assigned zero rainfall values, which are propagated through the morphing technique, thus introducing strong underestimations of precipitation accumulations. Future extensions of this study should focus on evaluating these surface effects using in situ meteorological and snow cover datasets. Furthermore, the value of higher-spatial-resolution satellite rainfall products (e.g., PERSIANN at $\sim 0.04^{\circ}$ and $\mathrm{CMORPH}$ at $\sim 0.08^{\circ}$ ) should be examined, particularly during the warm season convective events, and evaluated in terms of their error propagation in simulating the hydrologic response of mountainous basins.

Acknowledgments. This work was supported by NASA Precipitation Measurement Mission Award NNX07AE31G. Efthymios Nikolopoulos was supported by EU FP7 Marie Curie Actions IEF (Project PIEF-GA-2011-302720). We acknowledge and appreciate 
Roberto Dinale from the Province of Bolzano for making the gauge data available in this study.

\section{REFERENCES}

AghaKouchak, A., E. Habib, and A. Bárdossy, 2010: Modeling radar rainfall estimation uncertainties: Random error model. J. Hydrol. Eng., 15, 265-274, doi:10.1061/(ASCE)HE.1943-5584.0000185.

_- A. Behrangi, S. Sorooshian, K. Hsu, and E. Amitai, 2011: Evaluation of satellite-retrieved extreme precipitation rates across the central United States. J. Geophys. Res., 116, D02115, doi:10.1029/2010JD014741.

Ali, A., A. Amani, and T. Lebel, 2005: Rainfall estimation in the Sahel. Part II: Evaluation of rain gauge networks in the CILSS countries and objective intercomparison of rainfall products. J. Appl. Meteor., 44, 1707-1722, doi:10.1175/JAM2305.1.

Anagnostou, E. N., 2004: Overview of overland satellite rainfall estimation for hydro-meteorological applications. Surv. Geophys., 25, 511-537, doi:10.1007/s10712-004-5724-6.

—, M. N. Anagnostou, W. F. Krajewski, A. Kruger, and B. J. Miriovsky, 2004: High-resolution rainfall estimation from $\mathrm{X}$-band polarimetric radar measurements. J. Hydrometeor., 5, 110-128, doi:10.1175/1525-7541(2004)005<0110: HREFXP $>2.0 . \mathrm{CO} ; 2$.

— , V. Maggioni, E. I. Nikolopoulos, T. Meskele, F. Hossain, and A. Papadopoulos, 2010: Benchmarking high-resolution global satellite rainfall products to radar and rain-gauge rainfall estimates. IEEE Trans. Geosci. Remote Sens., 48, 1667-1683, doi:10.1109/TGRS.2009.2034736.

Arkin, P. A., and P. E. Ardanuy, 1989: Estimating climatic-scale precipitation from space: A review. J. Climate, 2, 1229-1238, doi:10.1175/1520-0442(1989)002<1229:ECSPFS > 2.0.CO;2.

Borga, M., E. N. Anagnostou, G. Blöschl, and J.-D. Creutin, 2010: Flash floods: Observations and analysis of hydrometeorological controls. J. Hydrol., 394, 1-3, doi:10.1016/ j.jhydrol.2010.07.048.

Ciach, G. J., W. F. Krajewski, and G. Villarini, 2007: Product-errordriven uncertainty model for probabilistic quantitative precipitation estimation with NEXRAD data.J. Hydrometeor., 8 , 1325-1347, doi:10.1175/2007JHM814.1.

Dinku, T., P. Ceccato, E. Grover-Kopec, M. Lemma, S. J. Connor, and C. F. Ropelewski, 2007: Validation of satellite rainfall products over East Africa's complex topography. Int. J. Remote Sens., 28, 1503-1526, doi:10.1080/01431160600954688.

Ebert, E. E., J. E. Janowiak, and C. Kidd, 2007: Comparison of near-real-time precipitation estimates from satellite observations and numerical models. Bull. Amer. Meteor. Soc., 88, 4764, doi:10.1175/BAMS-88-1-47.

Fleming, K., J. Awange, M. Kuhn, and W. Featherstone, 2011: Evaluating the TRMM 3B43 monthly precipitation product using gridded raingauge data over Australia. Aust. Meteor. Oceanogr. J., 61, 171-184. [Available online at www.bom.gov. au/amoj/docs/2011/fleming.pdf.]

Frei, C., and C. Schär, 1998: A precipitation climatology of the Alps from high-resolution rain-gauge observations. Int. J. Climatol., 18, 873-900, doi:10.1002/(SICI)1097-0088(19980630)18:8<873:: AID-JOC255>3.0.CO;2-9.

Germann, U., G. Galli, M. Boscacci, and M. Bolliger, 2006: Radar precipitation measurement in a mountainous region. Quart. J. Roy. Meteor. Soc., 132, 1669-1692, doi:10.1256/qj.05.190.

Hossain, F., and E. N. Anagnostou, 2006: Assessment of a multidimensional satellite rainfall error model for ensemble generation of satellite rainfall data. IEEE Geosci. Remote S., 3, 419-423, doi:10.1109/LGRS.2006.873686.

Huffman, G. J., and Coauthors, 2007: The TRMM Multisatellite Precipitation Analysis (TMPA): Quasi-global, multiyear, combined-sensor precipitation estimates at fine scales. $J$. Hydrometeor., 8, 38-55, doi:10.1175/JHM560.1.

Joyce, R. J., J. E. Janowiak, P. A. Arkin, and P. Xie, 2004: CMORPH: A method that produces global precipitation estimates from passive microwave and infrared data at high spatial and temporal resolution. J. Hydrometeor., 5, 487-503, doi:10.1175/1525-7541(2004)005<0487:CAMTPG >2.0.CO;2.

Kidd, C., D. R. Kniveton, M. C. Todd, and T. J. Bellerby, 2003: Satellite rainfall estimation using combined passive microwave and infrared algorithms. J. Hydrometeor., 4, 1088-1104, doi:10.1175/ 1525-7541(2003)004<1088:SREUCP $>2.0 . C O ; 2$.

Mei, Y., E. N. Anagnostou, D. Stampoulis, E. I. Nikolopoulos, M. Borga, and H. J. Vegara, 2014: Rainfall organization control on the flood response of mild-slope basins. J. Hydrol., 510, 565-577, doi:10.1016/j.jhydrol.2013.12.013.

Michaelides, S., V. Levizzani, E. N. Anagnostou, P. Bauer, T. Kasparis, and J. E. Lane, 2009: Precipitation science: Measurement, remote sensing, climatology and modeling. Atmos. Res., 94, 512-533, doi:10.1016/j.atmosres.2009.08.017.

Mishra, A. K., 2012: Application of merged precipitation estimation technique to study intense rainfall events over India and associated oceanic region. Atmos. Climate Sci., 2, 222-229, doi:10.4236/acs.2012.22023.

Nikolopoulos, E. I., E. N. Anagnostou, and M. Borga, 2013: Using high-resolution satellite rainfall products to simulate a major flash flood event in northern Italy. J. Hydrometeor., 14, 171185, doi:10.1175/JHM-D-12-09.1.

Norbiato, D., M. Borga, R. Merz, G. Blöschl, and A. Carton, 2009: Controls on event runoff coefficients in the eastern Italian Alps. J. Hydrol., 375, 312-325, doi:10.1016/j.jhydrol.2009.06.044.

Petty, G. W., and W. F. Krajewski, 1996: Satellite estimation of precipitation over land. Hydrol. Sci. J., 41, 433-451, doi:10.1080/02626669609491519.

Piccolo, F., and G. B. Chirico, 2005: Sampling errors in rainfall measurements by weather radar. Adv. Geosci., 2, 151-155, doi:10.5194/adgeo-2-151-2005.

Sapiano, M. R., and P. A. Arkin, 2009: An intercomparison and validation of high-resolution satellite precipitation estimates with 3-hourly gauge data. J. Hydrometeor., 10, 149-166, doi:10.1175/2008JHM1052.1.

Schneebeli, M., D. Nicholas, M. Lehning, and A. Berne, 2013: High-resolution vertical profiles of X-band polarimetric radar observables during snowfall in the Swiss Alps. J. Appl. Meteor. Climatol., 52, 378-394, doi:10.1175/JAMC-D-12-015.1.

Scofield, R. A., and R. J. Kuligowski, 2003: Status and outlook of operational satellite precipitation algorithms for extremeprecipitation events. Wea. Forecasting, 18, 1037-1051, doi:10.1175/1520-0434(2003)018<1037:SAOOOS > 2.0.CO;2.

Sharif, H. O., F. L. Ogden, W. F. Krajewski, and M. Xue, 2002: Numerical simulations of radar rainfall error propagation. Water Resour. Res., 38, 1140, doi:10.1029/2001WR000525.

Sorooshian, S., K.-L. Hsu, X. Gao, H. V. Gupta, B. Imam, and D. Braithwaite, 2000: Evaluation of PERSIANN system satellite-based estimates of tropical rainfall. Bull. Amer. Meteor. Soc., 81, 2035-2046, doi:10.1175/1520-0477(2000)081<2035: EOPSSE $>2.3 . \mathrm{CO} ; 2$.

Stampoulis, D., and E. N. Anagnostou, 2012: Evaluation of global satellite rainfall products over continental Europe. J. Hydrometeor., 13, 588-603, doi:10.1175/JHM-D-11-086.1. 
Steele, J. M., 2004: The Cauchy-Schwarz Master Class: An Introduction to the Art of Mathematical Inequalities. Cambridge University Press, 306 pp.

Su, F., Y. Hong, and D. P. Lettenmaier, 2008: Evaluation of TRMM Multisatellite Precipitation Analysis (TMPA) and its utility in hydrologic prediction in the La Plata basin. J. Hydrometeor., 9, 622-640, doi:10.1175/2007JHM944.1.

Tian, Y., and Coauthors, 2009: Component analysis of errors in satellite-based precipitation estimates. J. Geophys. Res., 114, D24101, doi:10.1029/2009JD011949.
Yong, B., L.-L. Ren, Y. Hong, J.-H. Wang, J. J. Gourley, S.-H. Jiang, X. Chen, and W. Wang, 2010: Hydrologic evaluation of Multisatellite Precipitation Analysis standard precipitation products in basins beyond its inclined latitude band: A case study in Laohahe basin, China. Water Resour. Res., 46, W07542, doi:10.1029/2009WR008965.

Zoccatelli, D., M. Borga, A. Viglione, G. B. Chirico, and G. Blöschl, 2011: Spatial moments of catchment rainfall: Rainfall spatial organisation, basin morphology, and flood response. Hydrol. Earth Syst. Sci., 15, 3767-3783, doi:10.5194/hess-15-3767-2011. 